\title{
Half-Wave Cylindrical Antenna in a Dissipative Medium: Current and Impedance
}

\author{
Ronold King ${ }^{2}$ and Charles W. Harrison ${ }^{3}$
}

\author{
(January 7, 1960)
}

\begin{abstract}
An integral equation for the distribution of current along a cylindrical antenna in a conducting dielectric is derived. It is shown that the boundary conditions for an antenna in such a medium are formally the same as for an antenna in free space. The equation is solved for the current $I$ and the driving-point impedance $Z$ by means of a technique that achieves sufficiently high accuracy in the leading terms of an iteration procedure so that the higher-order terms do not need to be evaluated. Moreover, these leading terms consist only of trigonometric functions with complex coefficients. The electromagnetic field in the infinite dissipative medium may be computed relatively easily since the current in the antenna is expressed in such simple terms.

A numerical analysis is made to determine the properties of an antenna with an electrical length of one-half wavelength in the medium with conductivity $\sigma$ and relative dielectric constant $\epsilon_{r}$. Universal curves are given of $I / \sqrt{\epsilon_{r}}$ with $\sigma / \omega \epsilon_{0} \epsilon_{r}$ as the parameter and of $Z \sqrt{\epsilon_{r}}$ with $\sigma / \omega \epsilon_{0} \epsilon_{r}$ as the variable in the range $0 \leq \sigma / \omega \epsilon_{0} \epsilon_{r} \leq 0.4$. A table of numerical values of the impedance is given for media such as an isotropic ionosphere, dry salt, dry earth, wet earth, and lake water.
\end{abstract}

\section{Introduction}

A study of the properties of antennas in dissipative media is an interesting theoretical problem that may have practical significance. For example, it appears to be possible to make certain diagnostic measurements in the area of fusion reactors that depend on a knowledge of the impedance of an antenna in an ionized medium. Specifically, it may be possible to determine ionization densities in high-temperature plasmas in this manner. In one phase of a contemplated field operation known as "Plowshare," it is expected that certain information concerning the operation of a nuclear device buried in a salt mine can be obtained with a low-frequency radar and a dipole antenna embedded in salt. Such measurements require a knowledge of the characteristics of antennas immersed in such a medium as well as of signal attenuation and dispersion.

A knowledge of the shift of impedance of a stub-type missile antenna as it enters an ionized cloud (such as the ionosphere, the aurora australis or borealis, or one of the Van Allen belts) makes it possible to determine transmission loss due to antenna mismatch.

Generally speaking, if the distribution of current along an antenna in a dissipative medium is known the input admittance is also available, and it is a straightforward process, at least in principle, to calculate the electromagnetic field at any point in the medium. From the field the signal attenuation in the medium may be determined. This is of importance in telemetry work, and also in the theory of communications between submerged submarines.

A theory is developed in this paper for a perfectly conducting, symmetrical, center-driven antenna of finite radius immersed in an infinite medium of moderate attenuation. Attention is directed specifically to half-wave antennas. For example, the present method of analysis permits the calculation of the impedance of this antenna when buried in "poor earth" for which the relative dielectric constant is $\epsilon_{r}=7$, and the conductivity is $\sigma=10^{-3} \mathrm{mho} / \mathrm{m}$. The theory also applies to a base-driven quarter-wave radiator oriented at right angles to an infinite perfectly conducting plane. It is of practical importance that the impedance of a stub-type

${ }^{1}$ Contribution from Sandia Corp., Albuquerque, N. Mex.

2 Consultant to the Sandia Corp. and Gordon McKay Professor of Applied Physies, Harvard University.

${ }^{3}$ Member of the Scientific Staff, Sandia Laboratory. 
antenna protruding from a missile differs negligibly from the impedance of the same antenna when mounted on an infinite ground plane, provided that the dimensions of the carrying vehicle are not too small in terms of the wavelength. Note also that the impedance of an antenna in a homogeneous dissipative medium of finite extent is essentially the same as in an infinite medium provided the boundary is sufficiently far removed from the antenna that the electromagnetic field at the interface is reduced to a small value owing to absorption.

\section{Basic Electromagnetic Theory}

Maxwell's equations in a homogeneous dissipative medium are

$$
\begin{aligned}
& \operatorname{curl} \mathbf{E}=-j \omega \mathbf{B} \\
& \operatorname{curl} \mathbf{B}=j \omega \mu \xi \mathbf{E}
\end{aligned}
$$

where $\mathbf{E}$ and $\mathbf{B}$ are the electric and magnetic vectors, respectively, and $\omega=2 \pi f$ is the radian frequency. The constants $\mu=1 / \nu ; \sigma$; and $\epsilon$ are the absolute permeability, conductivity, and dielectric constant of the medium; and $\xi$ is the complex dielectric factor given by

$$
\xi=\epsilon\left(1-j \frac{\sigma}{\omega \epsilon}\right)
$$

The suppressed time dependence is $\exp (j \omega t)$.

The vector potential $\mathbf{A}$ and scalar potential $\phi$ are defined by

$$
\begin{aligned}
\operatorname{curl} \mathbf{A} & =\mathbf{B} \\
\operatorname{div} \mathbf{A} & =-j \omega \mu \xi \phi \\
-\operatorname{grad} \boldsymbol{\phi} & =\mathbf{E}+j \omega \mathbf{A}
\end{aligned}
$$

where (5) is the Lorentz condition. The elimination of $\phi$ from (5) and (6) leads to the following relation for the electric field in terms of the vector potential:

$$
\mathbf{E}=-\frac{j}{\omega \mu \xi}\left(\operatorname{grad} \operatorname{div} \mathbf{A}+k^{2} \mathbf{A}\right)
$$

where

$$
k^{2}=\omega^{2} \mu \xi=(\beta-j \alpha)^{2} .
$$

The numerical evaluation of $k$ may be expedited by noting that if $p=\sigma / \omega \epsilon$,

$$
k=\beta-j \alpha=\omega \sqrt{\mu \epsilon}(\sqrt{1-j p})=\omega \sqrt{\mu \epsilon}\{f(p)-j g(p)\}
$$

where $f(p)=\cosh \left(\frac{1}{2} \sin h^{-1} p\right)$ and $g(p)=\sinh \left(\frac{1}{2} \sin h^{-1} p\right)$. Tables of the functions $f(p)$ and $g(p)$ are available in the literature [1]. ${ }^{4}$

When $p^{2}<<4, k=\beta-j \alpha \approx \omega \sqrt{\mu \epsilon}-j \frac{\sigma}{2} \sqrt{\mu / \epsilon}$. Note that in this case $p=2 \alpha / \beta=\frac{\sigma}{\omega \epsilon}, \alpha^{2} / \beta^{2}<<1$.

The boundary conditions between the homogeneous dissipative medium, region 1, and the perfectly conducting cylindrical antenna, region 2 , are

$$
\left.\begin{array}{l}
\epsilon_{1} \hat{n}_{1} \cdot \mathbf{E}_{1}=-\eta_{1 f}-\eta_{2 f} \\
\hat{n}_{1} \times \mathbf{E}_{1}=0 \\
\nu_{1} \hat{n}_{1} \times \mathbf{B}_{1}=\mathbf{K}_{2 f} \\
\hat{n}_{1} \times \mathbf{B}_{1}=0
\end{array}\right\}
$$

${ }^{4}$ Figures in brackets indicate the literature references at the end of this paper. 
since $\mathbf{E}_{2}=\mathbf{B}_{2}=0$ in the perfect conductor. $\hat{n}_{1}$ is a unit vector directed normally out of region $1, \eta_{1 f}+\eta_{2 f}$ is the total surface density of charge on the boundary, and $\mathbf{K}_{2 f}$ is the current density on the surface of the antenna.

A surface equation of continuity [2] that applies to the thin layer containing the surface charges and currents may be expressed as follows:

$$
\operatorname{div} \mathbf{K}_{2}+j \omega\left(\eta_{1 f}+\eta_{2 f}\right)-\hat{n}_{1} \cdot \sigma \mathbf{E}_{1}=0 .
$$

It may be satisfied in two parts. On the perfectly conducting surface,

$$
\operatorname{div} \mathbf{K}_{2 f}+j \omega \eta_{2 f}=0
$$

and on the adjacent surface of the dissipative medium,

$$
j \omega \eta_{1 f}-\hat{n}_{1} \cdot \sigma \mathbf{E}_{1}=0 .
$$

Thus $\eta_{2 f}$ is the surface density of charge associated with the surface density of current $\mathbf{K}_{2 f}$ along the perfect conductor, and $\eta_{1 f}$ is the surface density of charge related to the component of the volume density of current

$$
J_{1 f}=\sigma E_{1}
$$

that leaves the conductor normally to enter the medium in which it is immersed.

The substitution of the value of $\eta_{1 f}$ from (14) into (10) and (11) brings the boundary conditions into the following symmetrical form:

$$
\left.\begin{array}{l}
\xi \hat{n}_{1} \cdot \mathbf{E}_{1}=-\eta_{2 f} \\
\hat{n}_{1} \times \mathbf{E}_{1}=0 \\
\nu \hat{n}_{1} \times \mathbf{B}_{1}=-\mathbf{K}_{2 f} \\
\hat{n}_{1} \cdot \mathbf{B}_{1}=0 .
\end{array}\right\}
$$

\section{Vector Potential on the Surface of the Antenna}

Let the axis of the antenna (fig. 1) coincide with the $z$-axis of a cylindrical system of coordinates $r, \theta, z$. Let its ends be at $z= \pm h$; its cylindrical surface at $r=a$. It is assumed that

$$
\left.\begin{array}{c}
\beta a \ll 1 \\
a \ll h
\end{array}\right\}
$$

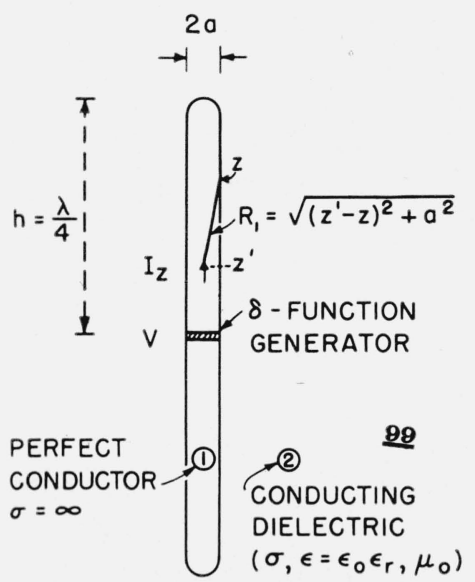

Figure 1. Half-wave dipole in a conducting medium. 
(where $\beta$ is defined by (9)). These conditions and an appropriate method of excitation that generates only a $z$-component of current in the antenna make it possible to set

$$
\mathbf{K}_{2 f}=\hat{z} K_{2 z} \text {. }
$$

The substitution of (4) in (17) with $\hat{n}_{1}=-\hat{r}$, gives

$$
\hat{n}_{1} \times \mathbf{B}_{1}=\nu \hat{n}_{1} \times \operatorname{curl} \mathbf{A}=\hat{\nu} \times \operatorname{curl} \mathbf{A}=-\hat{z} K_{2 z} .
$$

If $A_{r}=A_{\theta}=\frac{\partial A}{\partial \theta}=0,(20)$ becomes

$$
\hat{\nu r} \times \hat{\theta}\left(-\frac{\partial A_{z}}{\partial r}\right)=\hat{z} K_{2 z}
$$

or

$$
\left(\frac{\partial A_{z}}{\partial r}\right)_{r=a}=\left(\frac{\partial A_{z}}{\partial n}\right)_{r=a}=\frac{K_{2 z}}{\nu} .
$$

It is well known that the equation satisfied by the z-component of the vector potential in an infinite homogeneous dissipative medium is

$$
\nabla^{2} A_{z}+k^{2} A_{z}=0 .
$$

Its solution may be obtained by applying Green's theorem in the symmetric form,

$$
\int_{\tau}\left[u \nabla^{2} v-v \nabla^{2} u\right] d r^{\prime}=\int_{\Sigma}\left[u \frac{\partial v}{\partial n}-v \frac{\partial u}{\partial n}\right] d \sigma^{\prime}
$$

with $u=A_{z}$ and $v=G$, where $G$ is the free space Green's function that satisfies the auxiliary equation

$$
\nabla^{2} G+k^{2} G=-\delta\left(R_{d}\right) .
$$

In (25), $R_{d}=\left|\mathbf{R}-\mathbf{R}^{\prime}\right|$ is the distance between the point located by the end of the vector $\mathbf{R}^{\prime}$ where $G$ is calculated and a point source at the end of the vector $\mathbf{R}$. The Dirac delta function satisfies the relations

$$
\begin{aligned}
& \delta\left(R_{d}\right)=0, R_{d} \neq 0 \\
& \int_{\tau} \delta\left(R_{d}\right) d \tau\left\{\begin{array}{l}
1 \text { if } \tau \text { includes } R_{d}=0 \\
0 \text { if } \tau \text { excludes } R_{d}=0
\end{array}\right.
\end{aligned}
$$

The solution of (25) that vanishes at infinity is

$$
G=\frac{e^{-j k R_{d}}}{4 \pi R_{d}} .
$$

Equation 24 now becomes

$$
\int_{\tau}\left[G\left(\nabla^{2} A_{z}+k^{2} A_{z}\right)-A_{z}\left(\nabla^{2} G+k^{2} G\right)\right] d \tau^{\prime}=\int_{\Sigma_{1}+\Sigma_{2}}\left(G \frac{\partial A_{z}}{\partial n}-A_{z} \frac{\partial G}{\partial n}\right) d \sigma^{\prime}
$$

where $\Sigma_{2}$ is the surface of a great sphere, and $\Sigma_{1}$ is any parallel pair of surfaces within $\Sigma_{2}$ that enclose surfaces across which the functions $G$ and $A_{z}$ or their normal derivatives are discontinuous. In the case at hand the only such surface is the envelope of the perfectly conducting antenna of radius $a$, and the only one of the four functions that is discontinuous across it is $\partial A_{z} \mid \partial n$ as given in (22). It follows that with (23), (25), and (27) together with (22), (29) becomes

$$
\int_{\tau} A_{z} \delta\left(\mathbf{R}-\mathbf{R}^{\prime}\right) d \tau^{\prime}=\frac{1}{\nu} \int_{\Sigma_{1}} K_{2 z}\left(z^{\prime}\right) G d \sigma^{\prime}+\int_{\Sigma_{2}}\left(G \frac{\partial A_{z}}{\partial n}-A_{z} \frac{\partial G}{\partial n}\right) d \sigma^{\prime}
$$


The volume integral reduces to $A_{z}=A_{z}(\mathbf{R})$. Since $k$ is complex with negative imaginary part, both $\mathrm{A}_{z}$ and $G$ vanish at infinity. Accordingly, the integral over $\Sigma_{2}$ approaches zero as $\Sigma_{2}$ recedes to infinity. With $I_{z}\left(z^{\prime}\right)=2 \pi a K_{2 z}\left(z^{\prime}\right)$, (30) becomes

$$
A_{z}(\mathbf{R})=\frac{1}{4 \pi \nu} \int_{h}^{h} I_{z}\left(z^{\prime}\right) d z^{\prime} \int_{-\pi}^{\pi} \frac{e^{-j k R_{s}}}{R_{s}} \frac{d \theta^{\prime}}{2 \pi}
$$

where

$$
R_{s}=\sqrt{\left(z-z^{\prime}\right)^{2}+\left(2 a \sin \frac{\theta^{\prime}}{2}\right)^{2}}
$$

If $h \gg a$, as is assumed, an adequate approximation of (31) is

$$
A_{z}(\mathbf{B})=\frac{1}{4 \pi \nu} \int_{-h}^{h} I_{z}\left(z^{\prime}\right) \frac{e^{-j k R_{1}}}{R_{1}} d z^{\prime}
$$

where

$$
R_{1}=\sqrt{\left(z-z^{\prime}\right)^{2}+a^{2}} .
$$

The vector potential $A_{z}$ is defined in terms of the surface current $I_{z}=2 \pi a K_{2 z}$ on the perfect conductor. This current vanishes at the ends of the antenna if the end surfaces are neglected or if it is a tube and $I_{2}$ is the sum of the currents on inner and outer surfaces. That is

$$
I_{z}( \pm h)=0
$$

From (13), the surface current satisfies the equation of continuity

$$
\frac{d K_{2 z}}{d z}+j \omega \eta_{2 f}=0
$$

or when multiplied by $2 \pi a$,

$$
\frac{d I_{z}}{d z}+j \omega q_{2}=0
$$

where $q_{2}=2 \pi a \eta_{2 f}$ is the surface charge per unit length associated with the current along the conductor. The axial current is associated with $q_{2}$, and the radial diffusion current in the medium with $\eta_{1 f}$ in the equation of continuity

$$
j \omega \eta_{1 f}-\left(\sigma E_{1 \tau}\right)_{r=a}=0 .
$$

Thus, the physical picture is a sheet of current $I_{z}=2 \pi a K_{2 z}$ on the surface of the perfect conductor that is directed only axially and decreases by charging the surface. A portion of this charge remains for a part of the period in the axial standing wave, and a portion leaves radially into the adjacent imperfectly conducting or ionized medium. This is contained in the equation

$$
\frac{d I_{z}}{d z}+j \omega q_{2}+j \omega q_{1}-2 \pi a \sigma E_{1 r}=0
$$

where $q_{1}=2 \pi a \eta_{1 f}$.

\section{Integral Equation for the Current}

The integral equation for the current in a perfectly conducting antenna of length $2 h$ and radius $a$, center-driven at $z=0$ by an idealized delta-function generator with electromotive force $V$ may be derived easily from (23) and (33) with $\nabla^{2}=\partial^{2} / \partial z^{2}$. It is [3]

$$
\int_{-h}^{h} I_{z}\left(z^{\prime}\right) \frac{e^{-j k R_{1}}}{R_{1}} d z^{\prime}=-j \frac{4 \pi}{\zeta}\left\{C_{1} \cos k z+\frac{V}{2} \sin k|z|\right\} .
$$


In this relation $R_{1}=\sqrt{\left(z-z^{\prime}\right)^{2}+a^{2}}, C_{1}$ is a constant evaluated from the boundary condition (35), and

$$
\zeta=\sqrt{\frac{\mu}{\xi}}=\sqrt{\frac{j \omega \mu}{\sigma+j \omega \epsilon}} .
$$

Equation (40) is valid in an infinite homogeneous dissipative medium. In order that the present analysis may parallel a recent development in the theory of linear antenna arrays [4], an alternative form of (40) is desired. It is

$$
\int_{-h}^{h} I_{z}\left(z^{\prime}\right)\left\{\frac{e^{-j k R_{1}}}{R_{1}}-\frac{e^{-j k R_{h}}}{R_{h}}\right\} d z^{\prime}=-j \frac{4 \pi}{\zeta}\left\{C_{1} \cos k z+\frac{V}{2} \sin k|z|+U\right\}
$$

where

$$
U=-j \frac{\omega \mu}{4 \pi k} \int_{-h}^{h} I_{z}\left(z^{\prime}\right) \frac{e^{-j k R_{h}}}{R_{h}} d z^{\prime}=-j \frac{\omega}{k} A_{z}(h)
$$

and

$$
R_{h}=\sqrt{\left(h-z^{\prime}\right)^{2}+a^{2}}
$$

When $z=h$, (42) becomes

$$
C_{1} \cos k h+\frac{V}{2} \sin k h+U=0
$$

so that

$$
C_{1}=-\left\{\frac{\frac{1}{2} V \sin k h+U}{\cos k h}\right\}
$$

This value of $C_{1}$ may be substituted into (42) to obtain

$$
\int_{-h}^{h} I_{z}\left(z^{\prime}\right) K_{D}\left(z, z^{\prime}\right) d z^{\prime}=j \frac{4 \pi k}{\omega \mu \cos k h}\left\{\frac{1}{2} V \sin k(h-|z|)+U(\cos k z-\cos k h)\right\}
$$

where

$$
K_{D}\left(z, z^{\prime}\right)=\frac{e^{-j k R_{1}}}{R_{1}}-\frac{e^{-j k R_{h}}}{R_{h}}=e^{-\alpha R_{1}} \frac{e^{-j \beta R_{1}}}{R_{1}}-e^{-\alpha R_{h}} \frac{e^{-j \beta R_{h}}}{R_{h}} .
$$

Equation (46) is the integral equation for the current along a symmetrical center-driven antenna immersed in an infinite dissipative medium of arbitrary attenuation. The only restrictions that have been imposed are given by (18).

Since a delta-function generator is employed to drive the antenna, the driving point current, which defines the input admittance, is rigorously infinite because there is included an infinite admittance across the input terminals given by [5]

$$
Y=\underset{\infty}{G}+j \omega C=\frac{4 a}{\zeta} \ln \left\{\frac{1}{\left|z^{\prime}+\frac{h^{\prime}}{2}\right|}\right\}
$$

as $z^{\prime} \rightarrow-h^{\prime} / 2$ where $z^{\prime}=z-h, h^{\prime}=2 h$. The conductance and the capacitance are infinite since the knife edges between the idealized halves of the antenna are separated zero distance and are in contact with the dissipative medium. However, if (40) or (46) is solved in terms of continuous functions by the usual method of iteration, the driving point current actually obtained is essentially that maintained by the delta-function generator minus this infinite gap current. This is the desired approximate solution for the current in a practical antenna driven by an actual transmission line if suitable end corrections are provided. 


\section{Approximate Solution of the Integral Equation for the Current}

Let a solution of (46) be sought specifically for the half-wave dipole defined by $\beta h=\pi / 2$. The procedure followed is described in the literature [4]. In brief, it involves the separation of the total current along the antenna and the vector potential on its surface into several parts and the proper association of these to permit the introduction of several expansion functions that are sensibly constant along the antenna. Each of these functions is defined as the ratio of a particular component of the vector potential to its associated current. Several integral equations are obtained in this way which may be iterated independently of one another. It has been found that the sum of only the leading trigonometric terms of these component currents with suitably defined complex coefficients yields the total current and the driving point impedance with satisfactory accuracy.

The solution of (46) is begun by studying the variation with respect to $z$ of the functions [6]

$$
\Phi_{v}(z)=\int_{h}^{h} \sin k\left(h-\left|z^{\prime}\right|\right) K_{D}\left(z, z^{\prime}\right) d z^{\prime},
$$

and

$$
\Phi_{u}(z)=\int_{h}^{h}\left(\cos k z^{\prime}-\cos k h\right) K_{D}\left(z, z^{\prime}\right) d z^{\prime} .
$$

Now

$$
\sin k(h-|z|)=\cos \beta z \cosh \left\{\alpha h\left(1-\frac{|z|}{h}\right)\right\}-j \sin \beta|z| \sinh \left\{\alpha h\left(1-\frac{|z|}{h}\right)\right\}
$$

and

$$
\cos k z-\cos k h=\cos \beta z \cosh \left\{\alpha h \cdot \frac{z}{h}\right\}-i\left[\sinh \alpha h-\sin \beta z \beta z \sinh \left\{\alpha h \cdot \frac{z}{h}\right\}\right]
$$

when $\beta h=\pi / 2$. These quantities do not differ significantly in general form from those with $\alpha=0$, and the additional terms (which vanish when $\alpha=0$ ) are not sufficiently great to dominate provided the condition $\alpha h \leq 1$ is imposed. Actually, when $\alpha=\beta, \alpha h=1.57$. The slightly more severe condition,

$$
(\alpha h)^{2}<<2 \text { or } \alpha h \leq 0.3
$$

permits the following approximations:

$$
\cos h \alpha h \approx 1, \sin h \approx \alpha h, e^{-a R} \approx 1-\alpha R .
$$

It follows that

$$
\sin k(h-|z|) \approx \beta z-j \alpha h\left(1-\frac{|z|}{h}\right) \sin \beta|z|
$$

and

$$
\cos k z-\cos k h \approx \cos \beta z-j \alpha h\left(1-\frac{z}{h} \sin \beta z\right) .
$$

If the approximations

$$
\left(1-\frac{|z|}{h}\right) \sin \beta|z| \approx \cos \beta z+\sin \beta|z|-1
$$

and

$$
1-\frac{z}{h} \sin \beta z \approx \cos \beta z
$$

are made, it follows that

and

$$
\sin k(h-|z|) \approx(1-j \alpha h) \cos \beta z+j \alpha h(1-\sin \beta|z|)
$$

$$
\cos k z-\cos k h \approx(1-j a h) \cos \beta z .
$$


The approximations (57) and (58) are used throughout the analysis of the half-wave dipole antenna in a dissipative medium. They may be shown to be very good when $\beta h=\pi / 2$ by direct graphical comparison. Since $e^{-a R_{1}} \approx 1-\alpha R_{1}, K_{D}\left(z, z^{\prime}\right)$, as defined by (47), may be written

$$
K_{D}\left(z, z^{\prime}\right)=K_{d}\left(z, z^{\prime}\right)-\alpha K_{e}\left(z, z^{\prime}\right)
$$

where

$$
K_{d}\left(z, z^{\prime}\right)=\frac{e^{-j \beta R_{1}}}{R_{1}}-\frac{e^{-j \beta R_{h}}}{R_{h}}
$$

and

$$
K_{e}\left(z, z^{\prime}\right)=e^{-j \beta R_{1}}-e^{j \beta R_{h}}
$$

With (59) to (61), inclusive, (49) becomes

$$
\begin{aligned}
& \Phi_{v}(z) \approx(1-j \alpha h) \int_{h}^{h} \cos \beta z\left\{K_{d}\left(z, z^{\prime}\right)-\alpha K_{e}\left(z, z^{\prime}\right)\right\} d z^{\prime} \\
& +j \alpha h \int_{h}^{h}(1-\sin \beta|z|)\left\{K_{d}\left(z, z^{\prime}\right)-\alpha K_{e}\left(z, z^{\prime}\right)\right\} d z^{\prime} \\
& \begin{aligned}
\approx\left(1-j \frac{\alpha \lambda}{4}\right)\left\{C_{a}\left(\frac{\lambda}{4}, z\right)-C_{a}\left(\frac{\lambda}{4}, \frac{\lambda}{4}\right)-\frac{\alpha}{\beta}\left[\frac{\pi}{2}(1+j)-j\right] \cos \beta z\right\} \\
+j \alpha \frac{\lambda}{4}\left\{E_{a}\left(\frac{\lambda}{4}, z\right)-E_{a}\left(\frac{\lambda}{4}, \frac{\lambda}{4}\right)-S_{a}\left(\frac{\lambda}{4}, z\right)+S_{a}\left(\frac{\lambda}{4}, \frac{\lambda}{4}\right)\right\} .
\end{aligned}
\end{aligned}
$$

Terms of order $\alpha^{2} h / \beta$ and $\alpha^{2} h^{2}$ have been neglected. The particular values of the general functions $C_{a}, E_{a}$ and $S_{a}{ }^{5}$ required in this application are given later. In a similar manner (50) becomes

$$
\begin{gathered}
\Phi_{u}(z) \approx(1-j \alpha h)\left\{\int_{-h}^{h} \cos \beta z^{\prime} K_{d}\left(z, z^{\prime}\right) d z^{\prime}-\alpha \int_{-h}^{h} \cos \beta z^{\prime} K_{e}\left(z, z^{\prime}\right) d z^{\prime}\right\} \\
\approx\left(1-j \alpha \frac{\lambda}{4}\right)\left\{C_{a}\left(\frac{\lambda}{4}, z\right)-C_{a}\left(\frac{\lambda}{4}, \frac{\lambda}{4}\right)-\frac{\alpha}{\beta}\left[\frac{\pi}{2}(1+j)\right] \cos \beta z\right\} .
\end{gathered}
$$

It is known [4] that $\left.C_{a}(\lambda / 4, z)-C_{a} \lambda / 4, \lambda / 4\right)$ varies approximately as $\cos \beta z$ in both its real and imaginary parts. It follows that $\Phi_{u}(z)$ does also. Accordingly

$$
\Phi_{u}(z) \approx \Phi_{u}(0) \cos \beta z
$$

where

$$
\Phi_{u}=\Phi_{u}(0)=\left(1-j \alpha \frac{\lambda}{4}\right)\left\{C_{a}\left(\frac{\lambda}{4}, 0\right)-C_{a}\left(\frac{\lambda}{4}, \frac{\lambda}{4}\right)-\frac{\alpha}{\beta}\left[\frac{\pi}{2}(1+j)-j\right]\right\} .
$$

A comparison of (64) and (65) shows that

$$
\Phi_{v}(z) \approx \Phi_{u}(z)+\Phi_{w}(z)
$$

where

$$
\Phi_{w}(z)=j \alpha \frac{\lambda}{4}\left\{E_{a}\left(\frac{\lambda}{4}, z\right)-E_{a}\left(\frac{\lambda}{4}, \frac{\lambda}{4}\right)-S_{a}\left(\frac{\lambda}{4}, z\right)+S_{a}\left(\frac{\lambda}{4}, \frac{\lambda}{4}\right)\right\}
$$

\footnotetext{
${ }^{5}$ These functions $C_{a}(h, z)$ and $S_{a}(h, z)$ and $E_{a}(h, z)$ are defined as follows (see ref. [3], Sec. 19): $C_{a}(h, z)=\int_{0}^{h} L \cos \beta z^{\prime} d z^{\prime}, S_{a}(h, z)=\int_{0}^{h} L$ $\sin \beta z^{\prime} d z^{\prime}, E_{a}(h, z)=\int_{0}^{h} L d z^{\prime}$, where $L=\left\{\left(e^{-j \beta R_{1}} / R_{1}\right)+\left(e^{-\beta R_{2}} / R_{2}\right)\right\}, R_{1}=\sqrt{\left(z-z^{\prime}\right)^{2}+, a^{2}}, R_{2}=\sqrt{\left(z+z^{\prime}\right)^{2}+a^{2}}$. They may be expressed in terms of tabulated functions.
} 
Since it is readily verified that $\Phi_{\imath}(z)$ varies like $(1-\sin \beta|z|)$ in both its real and imaginary parts, it follows that

$$
\Phi_{v}(z) \approx \Phi_{u}(0) \cos \beta z+\Phi_{v}(0)(1-\sin \beta|z|) .
$$

In $(70)$,

$$
\Phi_{w}=\Phi_{w}(0)=j \alpha \frac{\lambda}{4}\left\{E_{a}\left(\frac{\lambda}{4}, 0\right)-E_{a}\left(\frac{\lambda}{4}, \frac{\lambda}{4}\right)-S_{a}\left(\frac{\lambda}{4}, 0\right)+S_{a}\left(\frac{\lambda}{4}, \frac{\lambda}{4}\right)\right\}
$$

From $(68)$ or $(70)$,

$$
\Phi_{v} \approx \Phi_{u}+\Phi_{w} .
$$

This completes the investigation of the functions $\Phi_{v}(z)$ and $\Phi_{u}(z)$.

With (59) to (61), and the approximation $\cos k h \approx j \alpha h$, (46) may be expressed as follows:

$$
\begin{gathered}
\int_{-h}^{h} I_{v 1}\left(z^{\prime}\right)\left\{K_{d}\left(z, z^{\prime}\right)-\alpha K_{e}\left(z, z^{\prime}\right)\right\} d z^{\prime}+\int_{-h}^{h} I_{v 2}\left(z^{\prime}\right)\left\{K_{d}\left(z, z^{\prime}\right)-a K_{e}\left(z, z^{\prime}\right)\right\} d z^{\prime}+\int_{-h}^{h} I_{u}\left(z^{\prime}\right)\left\{K_{d}\left(z, z^{\prime}\right)\right. \\
\left.-\alpha K_{e}\left(z, z^{\prime}\right)\right\} d z^{\prime}=\frac{4 \pi(\beta-j \alpha)}{\omega \mu \alpha h}\left\{\left[\frac{1}{2} V+U\right][1-j \alpha h] \cos \beta z+j \frac{1}{2} V \alpha h(1-\sin \beta|z|)\right\}
\end{gathered}
$$

where $I_{z}\left(z^{\prime}\right)$ has been replaced by

$$
I_{z}\left(z^{\prime}\right)=I_{v 1}\left(z^{\prime}\right)+I_{v 2}\left(z^{\prime}\right)+I_{u}\left(z^{\prime}\right) .
$$

The integrals in (73) may be written as follows:

where

$$
\left.\begin{array}{l}
\int_{-h}^{h} I_{v 1}\left(z^{\prime}\right)\left\{K_{d}\left(z, z^{\prime}\right)-\alpha K_{e}\left(z, z^{\prime}\right)\right\} d z^{\prime}=\psi_{v 1} I_{v 1}(z)-D_{v 1}(z) \\
\int_{-h}^{h} I_{v 2}\left(z^{\prime}\right)\left\{K_{d}\left(z, z^{\prime}\right)-\alpha K_{e}\left(z, z^{\prime}\right)\right\} d z^{\prime}=\psi_{v 2} I_{v 2}(z)-D_{v 2}(z) \\
\int_{-h}^{h} I_{u}\left(z^{\prime}\right)\left\{K_{d}\left(z, z^{\prime}\right)-\alpha K_{e}\left(z, z^{\prime}\right)\right\} d z^{\prime}=\psi_{u} I_{u}(z)-D_{u}(z)
\end{array}\right\}
$$

and

$$
\psi_{i}=\int_{-h}^{h} g_{i}\left(z, z^{\prime}\right)\left\{K_{d}\left(z, z^{\prime}\right)-\alpha K_{e}\left(z, z^{\prime}\right)\right\} d z^{\prime}
$$

$$
D_{i}=\int_{-h}^{h}\left[I_{i}\left(z^{\prime}\right)-I_{i}(z) g_{i}\left(z, z^{\prime}\right)\right]\left[K_{d}\left(z, z^{\prime}\right)-\alpha K_{e}\left(z, z^{\prime}\right)\right] d z^{\prime}
$$

where the subscript $i$ stands for $v_{1}, v_{2}$, or $u$.

An inspection of (73) and the $\Phi(z)$ functions previously studied, shows that a proper association of the components of the vector potential and the current is achieved if the following choice of distribution functions is made:

and

$$
\left.\begin{array}{l}
g_{v 1}\left(z, z^{\prime}\right)=g_{u}\left(z, z^{\prime}\right)=\frac{\cos \beta z^{\prime}}{\cos \beta z} \\
g_{v 2}\left(z, z^{\prime}\right)=\frac{1-\sin \beta\left|z^{\prime}\right|}{1-\sin \beta|z|}
\end{array}\right\} .
$$

Then, with (76),

and

$$
\left.\begin{array}{l}
\psi_{v 1}=\psi_{u}=\frac{\Phi_{u}}{1-j \alpha h} \\
\psi_{v 2}=\frac{\Phi_{w}}{j \alpha h}
\end{array}\right\} .
$$


This pairing of vector potentials and currents insures that to a high degree of approximation. $\psi_{v 1} \sim A_{v 1}(z) / I_{v 1}(z), \psi_{v 2} \sim A_{v 2}(z) / I_{v 2}(z)$, and $\psi_{u} \sim A_{u}(z) / I_{u}(z) . \quad A_{v 1}(z), A_{v 2}(z)$, and $A_{u}(z)$ are components of the vector potential calculated from the currents $I_{v 1}(z), I_{v 2}(z)$, and $I_{u}(z)$, respectively. It follows that all difference integrals, defined by (77), are small.

The substitution of (75) into (73) gives

$$
\begin{aligned}
I_{v 1}(z) \psi_{v 1}+I_{v 2}(z) \psi_{v 2}+I_{u}(z) \psi_{u}=\frac{4 \pi(\beta-j \alpha)}{\omega \mu \alpha h}\{ & \left(\frac{1}{2} V+U\right)(1-j \alpha h) \cos \beta z \\
& \left.+j \frac{1}{2} V \alpha h(1-\sin \beta|z|)\right\}+D_{v 1}(z)+D_{v 2}(z)+D_{u}(z) .
\end{aligned}
$$

Since $\psi_{v 1}=\psi_{u}$ and the components of the vector potential contributed by $I_{v 1}(z)$ and $I_{u}(z)$ vary as $\cos \beta z$, (80) may be separated into two parts and these iterated separately. Thus

$$
\begin{gathered}
\left.I_{v 1}(z)+I_{u}(z)=\frac{4 \pi(\beta-j \alpha)}{\omega \mu \alpha h \psi_{u}}\left\{\frac{1}{2} V+U\right)(1-j \alpha h) \cos \beta z\right\}+\frac{1}{\psi_{u}}\left\{D_{v 1}(z)+D_{u}(z)\right\} . \\
I_{v 2}(z)=j \frac{4 \pi(\beta-j \alpha)}{\omega \mu \alpha h \psi_{v 2}}\left\{\frac{1}{2} V \alpha h(1-\sin \beta|z|)+\frac{D_{v 2}(z)}{\psi_{v 2}}\right\} .
\end{gathered}
$$

The leading terms may be substituted into the difference integrals to obtain first order corrections to the currents, if desired.

The total current is

$$
I_{z}(z)=\frac{2 \pi(\beta-j \alpha) V}{\omega \mu \alpha h \psi_{u}}\left\{\left(1+\frac{2 U}{V}\right)(1-j \alpha h) \cos \beta z+j \alpha h(1-\sin \beta|z|) \frac{\psi_{u}}{\psi_{v 2}}+\ldots\right\} .
$$

The ratio $2 U / V$ may be determined from (43) with the substitution of $I_{z}(z)$ as given by (83), i.e.,

$$
\begin{aligned}
U=-j \frac{\omega \mu}{4 \pi(\beta-j \alpha)} \int_{-h}^{h} I_{z}\left(z^{\prime}\right)\left\{\frac{e^{-j \beta R_{1 h}}}{R_{1 h}}-\alpha e^{-j \beta R_{1 h}}\right\} d z^{\prime} \\
=-j \frac{V}{2 \alpha h \psi_{u}}\left\{\left(1+\frac{2 U}{V}\right)(1-j \alpha h) \psi_{c}(h)+j \alpha h \frac{\psi_{s}(h) \psi_{u}}{\psi_{v 2}}\right\}
\end{aligned}
$$

where

and

$$
\psi_{c}(h)=\int_{-h}^{h} \cos \beta z^{\prime}\left\{\frac{e^{-j \beta R_{1 h}}}{R_{1 h}}-\alpha e^{-j \beta R_{1 h}}\right\} d z^{\prime} \approx C_{a}\left(\frac{\lambda}{4}, \frac{\lambda}{4}\right)+j \alpha h
$$

$$
\psi_{s}(h)=\int_{-h}^{h}\left(1-\sin \beta\left|z^{\prime}\right|\right)\left\{\frac{e^{-j \beta R_{1 h}}}{R_{1 h}}-\alpha e^{-j \beta R_{1 h}}\right\} d z^{\prime} \approx E_{a}\left(\frac{\lambda}{4}, \frac{\lambda}{4}\right)-S_{a}\left(\frac{\lambda}{4}, \frac{\lambda}{4}\right)+j \frac{\alpha}{\beta} .
$$

The solution of (84) for $2 U / V$ yields

$$
\frac{2 U}{V}=\frac{\frac{\psi_{s}(h) \psi_{u}}{\psi_{v 2}}-\left(1+\frac{j}{\alpha h}\right) \psi_{c}(h)}{\psi_{u}+\psi_{c}(h)\left(1+\frac{j}{\alpha h}\right)} .
$$

The substitution of (87) into (83) gives the following final expression for the leading terms of the current:

$$
I_{z}(z)=-j \frac{2 \pi(\beta-j \alpha) V}{\omega \mu \psi_{v 2}}\left\{T\left(\frac{\lambda}{4}\right) \cos \beta z+\sin \beta|z|-1\right\}
$$

The corresponding driving point impedance is 


$$
Z(k)=\frac{V}{I_{z}(0)}=j \frac{\omega \mu \psi_{v 2}}{2 \pi(\beta-j \alpha)\left\{T\left(\frac{\lambda}{4}\right)-1\right\}} .
$$

In (88) and (89),

$$
T\left(\frac{\lambda}{4}\right)=\frac{\left(1-j \frac{\pi}{2} \frac{\alpha}{\beta}\right)\left(\psi_{v 2}+\psi_{s}\left(\frac{\lambda}{4}\right)\right)}{\left(1-j \frac{\pi}{2} \frac{\alpha}{\beta}\right) \psi_{c}\left(\frac{\lambda}{4}\right)-j \frac{\pi}{2} \frac{\alpha}{\beta} \psi_{u}} .
$$

The use of approximte forms of the $C_{a}, S_{a}$, and $E_{a}$ functions [6], leads to the following formulas. The final numerical results in (91) apply to a half-wave dipole with $h / a=75$ or $a / \lambda=1 / 300$.

$$
\begin{aligned}
\psi_{u} & =2 \sinh ^{-1} \frac{h}{a}-2.357-j 0.633-\frac{\alpha}{\beta}(1.571+j 0.571) \\
& =7.663-j 0.633-\frac{\alpha}{\beta}\left(1.571+j 0.571 \quad \text { for } \frac{h}{a}=75\right. \\
\psi_{v 2} & =4 \sinh ^{-1} \frac{h}{a}-2 \sinh ^{-1} \frac{2 h}{a}-1.747-j 0.384 \\
& =6.885-j 0.384 \quad \text { for } \frac{h}{a}=75 \\
\psi_{c} & =0.709-j 1.219+j \frac{\pi \alpha}{2 \beta} \quad \text { for } \frac{h}{a}=75 . \\
\psi_{s} & =2 \sinh ^{-1} \frac{2 h}{a}-2 \sinh ^{-1} \frac{h}{a}-1.219-j 0.709+j \frac{\alpha}{\beta} \\
& =0.167-j 0.709+j \frac{\alpha}{\beta} \quad \text {. }
\end{aligned}
$$

\section{Distribution of Current}

The current as given in (88) involves the parameter $\beta / \omega \mu=\left(1 / \zeta_{0}\right) \sqrt{\epsilon_{r} / \mu_{r}} f(p)$ and the dimensionless ratio $p=2 \alpha / \beta=\sigma / \omega \epsilon$ that characterize the properties of the medium in which the antenna is immersed. If attention is restricted to nonmagnetic materials and values of $p \leq 0.5$, it follows that $f(p) \approx 1^{6}$ so that $\beta / \omega \mu=\sqrt{\epsilon_{r}} / \zeta_{0}$. In this case the two relevant quantities are $\sqrt{\epsilon_{r}}$ and $\sigma / \omega \epsilon_{0} \epsilon_{r}$. It follows that a normalized current may be defined that is a function of $2 \alpha / \beta$ or of $\sigma / \omega \epsilon_{0} \epsilon_{r}$ alone insofar as the properties of the medium are concerned. That is,

$$
\frac{I_{z}}{V \sqrt{\epsilon_{r}}}=\frac{-j 2 \pi\left(1-j \frac{\alpha}{\beta}\right)}{\zeta_{0} \psi_{v 2}}\left\{T\left(\frac{\lambda}{4}\right) \cos \beta z+\sin \beta|z|-1\right\}
$$

where $\zeta_{0}=\sqrt{\mu_{0} / \epsilon_{0}} \approx 120 \pi$ ohms and it is assumed that $\sigma / \omega \epsilon_{0} \epsilon_{r} \leq 0.5$. With the current expressed in the form $I_{z}=I_{z}^{\prime \prime}+j I_{z}^{\prime}=I_{z} \epsilon^{j \theta_{I}}$ the family of curves shown in figure 2 has been evaluated from (95) for the distribution of the real and imaginary parts of the normalized current along a half-wave dipole. The parameter is $\sigma / \omega \epsilon_{0} \epsilon_{r}$. The corresponding curves of the amplitude and phase are shown in figure 3. Note that these curves apply to an antenna for which $h / a=75$ and $\beta h=\pi / 2$ with $\beta=\omega \sqrt{\mu \epsilon}$ the phase constant appropriate to the dielectric medium. The actual

\footnotetext{
${ }^{6}$ When $p=0.5, f(p)=1.029$ which differs from unity by less than 3 percent.
} 

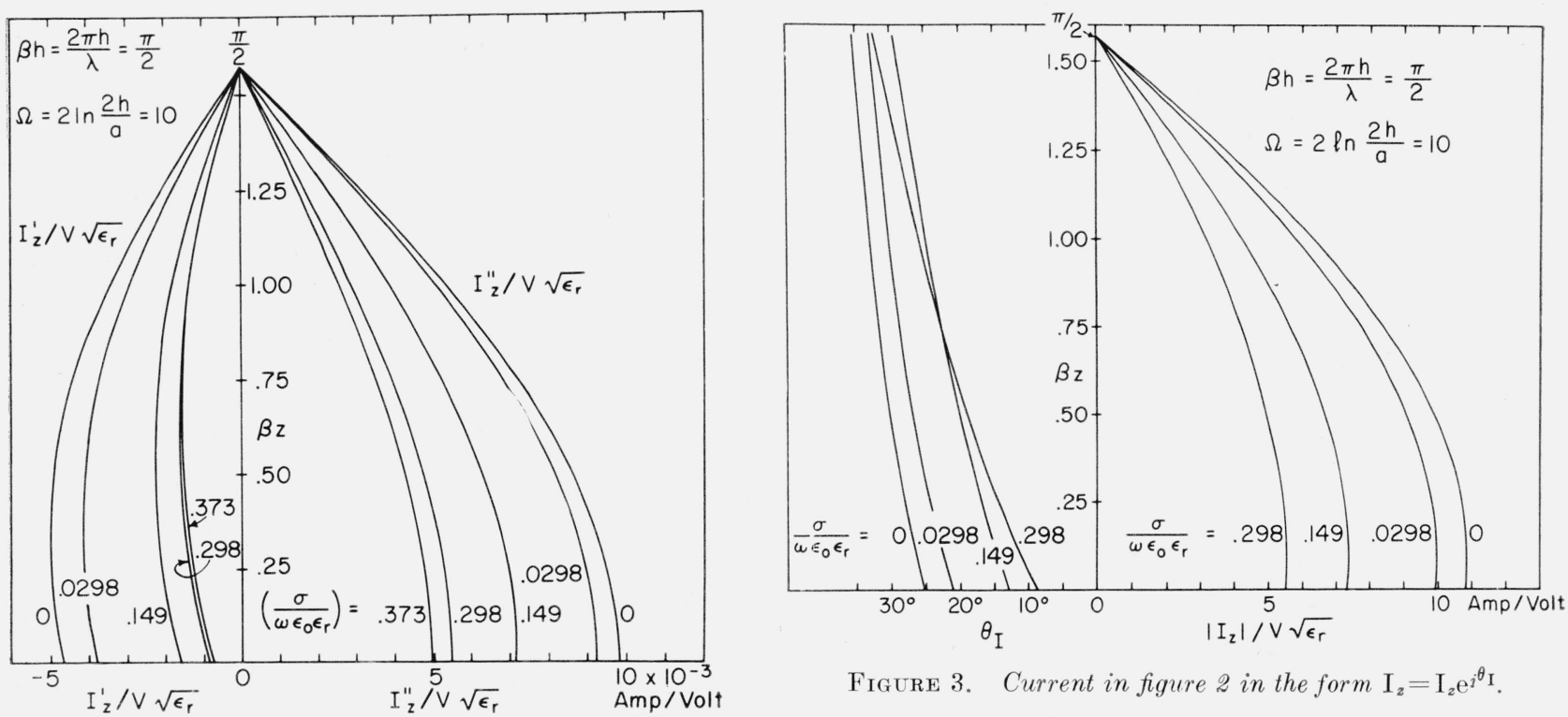

Figure 3. Current in figure 2 in the form $\mathrm{I}_{z}=\mathrm{I}_{2} \mathrm{e}^{i^{\theta} \mathrm{I}}$.

Figure 2. Current $\mathrm{I}_{\mathrm{z}}=\mathrm{I}_{\mathrm{z}}^{\prime \prime}+\mathrm{jI}_{\mathrm{z}}^{\prime}$ in normalized form for a half-wave dipole in a medium with conductivity $\sigma$ and dielectric constant $\epsilon=\epsilon_{0} \epsilon_{r} ; I_{z}^{\prime \prime}$ is in phase with the driving voltage $\mathrm{V}$.

currents per input volt are obtained from these figures by multiplying the normalized values with $\sqrt{\epsilon_{r}}$. The curves indicate that when an antenna is immersed in a medium of given dielectric constant, the amplitude of the current diminishes with increasing conductivity of the medium if the same driving voltage is applied. The inductive lag of the input current also decreases as $\sigma$ is made greater, but there is a rise in the relative phase change outward along the antenna. That is, the current near the end of the antenna lags behind the current at the driving point by an angle that increases with $\sigma$.

\section{Admittance and Impedance}

The admittance, $Y=G+j B$, and the impedance, $Z=R+j X$, are most conveniently expressed in the normalized forms, $Y / \sqrt{\epsilon_{r}}$ and $Z \sqrt{\epsilon_{r}}$. The former is obtained directly from (95) with $z=0$; evidently, $Z \sqrt{\epsilon_{r}}=1 /\left(Y \sqrt{\epsilon_{r}}\right)$. The normalized quantities, $G / \sqrt{\epsilon_{r}},-B / \sqrt{\epsilon_{r}}$ and $R \sqrt{\epsilon_{r}}$, $X \sqrt{\epsilon_{r}}$ are shown graphically in figure 4. In the range $\left(\sigma / \omega \epsilon_{0} \epsilon_{r}\right) \leq 0.5$ a single curve is obtained for each of these quantities for all values of $\sigma$ and $\epsilon_{r}$. It is seen that when an antenna is immersed in a medium with a given dielectric constant for which $2 h=\lambda / 2$ in the medium, its input resistance increases almost linearly with increasing conductivity of the medium while its reactance first decreases to a shallow minimum, then increases slightly. The actual admittance of a half-wave antenna when immersed in a medium with a given dielectric constant is obtained from figure 4 by multiplying the normalized conductance and susceptance by $\sqrt{\epsilon_{r}}$. Similarly, the impedance is obtained from the normalized resistance and reactance when divided by $\sqrt{\epsilon_{r}}$. Numerical values of actual resistances and reactances for a range of dielectric constants and conductivities are listed in the table 1 .

It is important to bear in mind that the data contained in figure 4 and table 1 always apply to an antenna that is exactly a half wavelength long in the medium with the specified dielectric constant and conductivity. That is, the electrical half-length is $\beta h=\pi / 2$. Note, however, that if an antenna has the half-length $h=\lambda / 4$ referred to the wavelength in the medium, its half-length referred to free space is $h=\lambda_{0} / 4 \sqrt{\epsilon_{r}}$. In other words, the electrical length $\beta_{0} h$ in free space corresponding to an electrical length $\beta h=\pi / 2$ in the medium is $\beta_{0} h=\beta h / \sqrt{\epsilon_{r}}$ when $\mu=\mu_{0}$. If an antenna that is adjusted in length so that $\beta h=\pi / 2$ in a medium with constants 


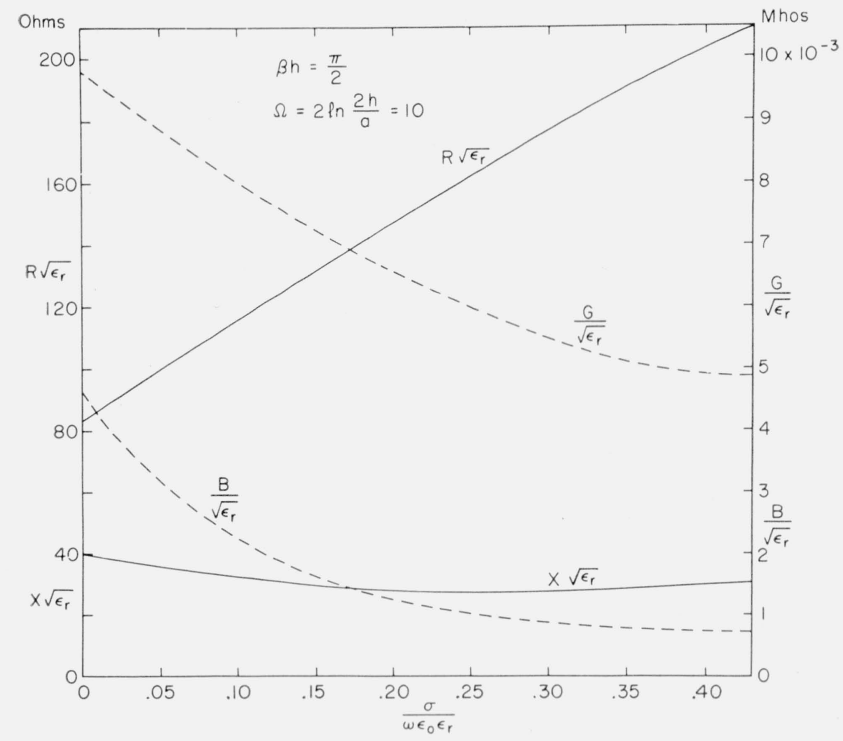

FIGURE 4. Impedance and admittance of a half-wave dipole when immersed in a medium with conductivity $\sigma$ and dielectric constant $\epsilon=\epsilon_{0} \epsilon_{r} ; \beta=\omega \sqrt{\mu \epsilon}=2 \pi / \lambda$.
TABLE 1. Impedances of antennas for which $\beta \mathrm{h}=\pi / 2$ when immersed in a dissipative medium; $\mathrm{h} / \mathrm{a}=75, \mathrm{f}=6 \mathrm{Mc}$

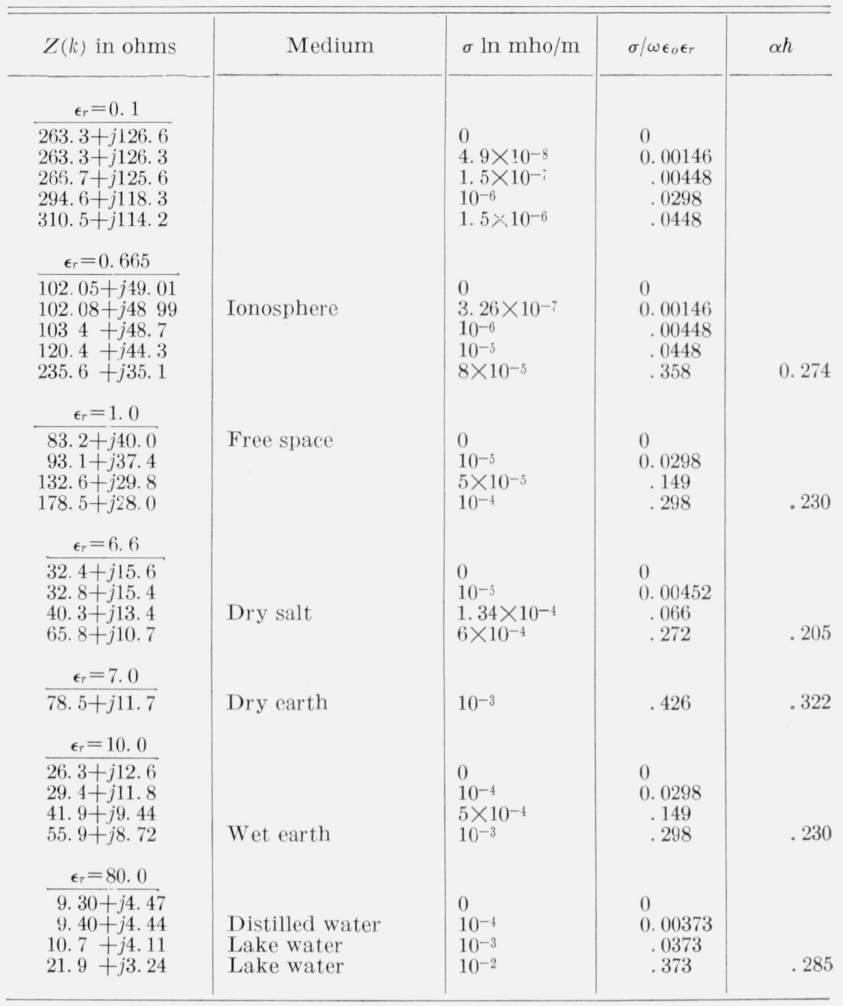

$\epsilon_{r}$ and $\sigma$ is removed to free space, its impedance changes both because the parameters that characterize the medium are different and because its electrical length is changed. This is illustrated in figure 5 which gives $R$ and $X$ for an antenna that has been removed to free space from a medium with relative dielectric constant $\epsilon_{r}$ in which its electrical length was $\pi / 2$.

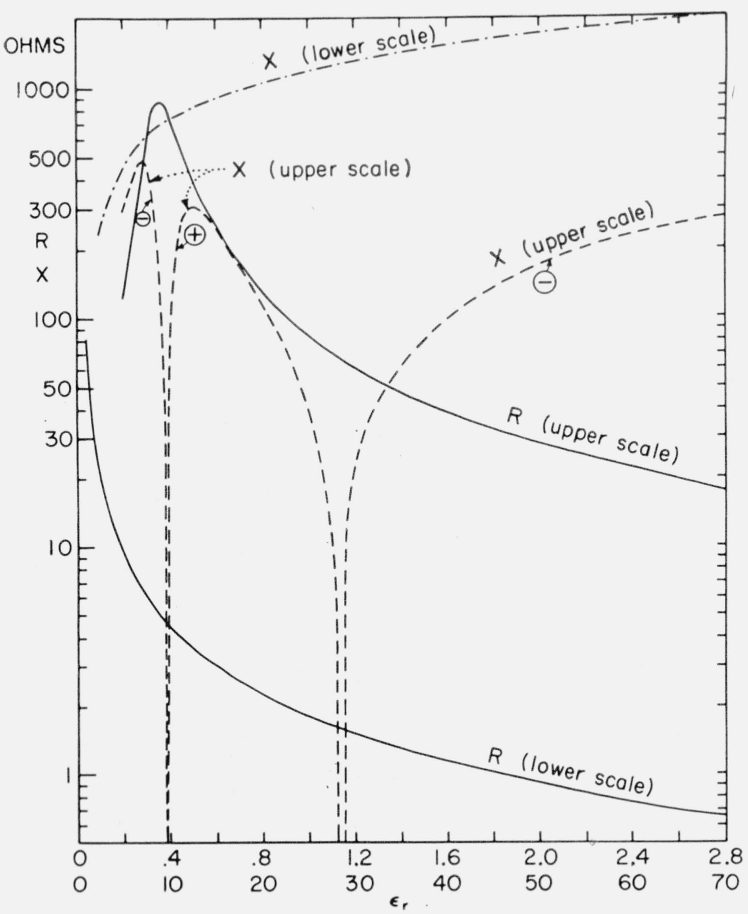

Figure 5. $\mathrm{R}$ and $\mathrm{X}$ of dipole in free space with halflength $\mathrm{h}$ (for which $\beta \mathrm{h}=\omega \mathrm{h} \sqrt{\mu_{0} \epsilon_{0} \epsilon_{r}}$ when the antenna is immersed in a dielectric with relative dielectric constant $\epsilon_{r}$ so that $\left.\mathrm{h}=\frac{\lambda_{0}}{4 \sqrt{\epsilon_{r}}}\right)$. 


\section{Special Cases}

A number of special cases are of interest. The following data apply to an antenna for which $\beta h=\pi / 2$ in the specified medium and $h / a=75$.

Case I. Antenna in free space. $\epsilon_{r}=1, \sigma=0$, so that $\alpha=0$ and $\beta=\beta_{0}=2 \pi / \lambda_{0}$.

$$
Z\left(\beta_{0}\right)=83.2+j 40.0 \text { ohms. }
$$

The King-Middleton second order theory gives [7]

$$
Z\left(\beta_{0}\right)=86.5+j 41.7 \mathrm{ohms} .
$$

It is noteworthy that the new approach to the analysis of cylindrical antennas [4] yields results that agree so closely with those of a complicated, twice-iterated solution (that is known to be in good agreement with experiment when proper account is taken of transmission-line end and coupling effects [8]), since only the leading trigonometric terms in the current distribution are used in obtaining (96a). This is a consequence of properly choosing the trigonometric functions and carefully adjusting their relative complex values.

Case II. Split missile sounding rocket. According to Nicolet [9] at an altitude of $100 \mathrm{~m}$ (where the temperature is $210^{\circ} \mathrm{K}$ ) the maximum collision frequency $\nu^{7}=1.1 \times 10^{5} \mathrm{sec}^{-1}$. At this same altitude, DiTaranto and Lamb [10] anticipate an electron density $N=1.5 \times 10^{11}$ electrons $\mathrm{m}^{3}$. The standard relations for the dielectric constant and conductivity of the ionosphere, neglecting the earth's magnetic field are

$$
\epsilon=\epsilon_{0}\left\{1-\frac{\mathrm{Ne}^{2}}{\epsilon_{0} m\left(\nu^{2}+\omega^{2}\right)}\right\}
$$

and

$$
\sigma=\frac{\mathrm{Ne}^{2} \nu}{m\left(\nu^{2}+\omega^{2}\right)}
$$

where $\epsilon_{0}=8.85 \times 10^{-12} \mathrm{f} / \mathrm{m}, e$ is the charge of the electron $\left(e=-1.60 \times 10^{-19}\right.$ coulomb), and $m$ is the mass of the electron $\left(m=9.11 \times 10^{-31} \mathrm{~kg}\right)$. With the above values for $N$ and $\nu$, a frequency of $6 \mathrm{Mc}, \epsilon=0.6649 \epsilon_{0}$ and $\sigma=3.26 \times 10^{-7} \mathrm{mho} / \mathrm{m}$, the attenuation constant of the medium is $\alpha=7.534 \times 10^{-5}$ nepers $/ \mathrm{m}$ and the phase constant is $\beta=0.1025 \mathrm{radians} / \mathrm{m}$. The impedance of the center-driven half-wave dipole in this medium is

$$
Z(k)=102.1+j 49.0 \mathrm{ohms} .
$$

In air the electrical half-length of this antenna is $\beta_{0} h=1.926$, its actual half length $h=15.3 \mathrm{~m}$, and its impedance is $Z\left(\beta_{0}\right)=200+j 203$ ohms. On comparing $Z(k)$ with $Z\left(\beta_{0}\right)$, it is clear that the electron density of the normal ionosphere can easily be determined with a half-wave dipole antenna used as a probe at $6 \mathrm{Mc}$.

Case III. Antenna in a Plasma. If the electron density is $4.05 \times 10^{11}$ electrons $/ \mathrm{m}^{3}$ and the collision frequency is $\nu=6.15 \times 10^{3} \mathrm{sec}^{-1}$ it follows that $\epsilon=0.1 \epsilon_{0}, \quad \sigma=4.9 \times 10^{-8} \mathrm{mho} / \mathrm{m}$, $\beta=0.040$ radians $/ \mathrm{m}, \alpha=2.92 \times 10^{-3}$ nepers $/ \mathrm{m}$, and the impedance of the half-wave dipole is

$$
Z(k)=263.3+j 126.3 \text { ohms. }
$$

The half-length of the antenna at $f=6 \mathrm{Mc}$ is $h=\pi / 2 \beta=39.3 \mathrm{~m}$.

Case IV. Antenna Buried in Salt. The measured properties of the salt at $6 \mathrm{Mc}$ are $\boldsymbol{\epsilon}_{r}=6.6$ and $\sigma=1.34 \times 10^{-4} \mathrm{mho} / \mathrm{m}$. It follows that $\beta=0.323 \mathrm{radians} / \mathrm{m}, \alpha=9.85 \times 10^{-3}$ nepers/ $\mathrm{m}$, and the impedance of the half-wave dipole is

$$
Z(k)=40.3+j 13.5 \text { ohms. }
$$

${ }^{7} \nu$ should not be confused with $\nu=1 / \mu . \quad \nu$ is retained as the symbol for collision frequency in order to agree with standard notation. 
Since

$$
\beta h=\frac{\pi}{2}, h=\frac{\pi}{2 \beta}=\frac{\pi}{2 \times 0.323}=4.86 \mathrm{~m}
$$

Case V. Antenna in "poor earth" for which $\boldsymbol{\epsilon}_{r}=7$ and $\sigma=10^{-3} \mathrm{mho} / \mathrm{m}$. At $6 \mathrm{Mc}, \beta=0.34$ radians $/ m$, and $\alpha=0.0697$ nepers $/ m$, and

$$
Z(k)=78.47+j 11.66 \text { ohms } .
$$

It should be mentioned that in this case $\alpha h=\alpha \pi / 2 \beta=0.322$. This value of $\alpha h$ is near the limit of validity of the approximations introduced in the analysis and contained in the condition $\alpha h \leq 0.3$.

Case VI. Antenna in a highly conducting medium. If the antenna is immersed in a highly conducting medium, the formula (88) for the distribution of current does not apply owing to the restriction $\alpha h \leq 0.3$. However, irrespective of the precise form of the distribution of current along the antenna, the formula (89) for the impedance should approach the correct limit, $Z(k) \rightarrow 0$, as $\sigma \rightarrow \infty$. When $\sigma / \omega \epsilon$ is large compared with unity

$$
\beta-j \alpha=(1-j) \sqrt{\frac{\omega \sigma \mu}{2}}
$$

as $\sigma \rightarrow \infty, \beta=\alpha \rightarrow \infty$, but $\alpha / \beta=1$. An examination of the $\psi_{u}, \psi_{v 2}, \psi_{c}$, and $\psi_{s}$ functions shows that they remain finite. Since $\beta-j \alpha$ appears in the denominator of (89), and all other functions are well behaved, $Z(k) \rightarrow 0$ as $\sigma \rightarrow \infty$.

\section{Conclusion}

Although the present analysis has been applied specifically to an antenna that is a half wavelength long when immersed in a conducting dielectric, and the conductivity of the dielectric has been limited to moderate values by the condition $\alpha h \leq 0.3$, the method is applicable to other lengths and higher conductivities. A study of antennas for which $\alpha h \leq 0.3$ but $\beta h=\pi$ is in progress. The electrically short antenna defined by $\beta h<1$ is also under investigation.

Since the properties of a two-wire line immersed in a conducting dielectric are understood, the extension of the present study to the folded dipole is readily accomplished with the method of symmetrical components. Details are reserved for a subsequent paper.

\section{References}

]1] R. W. P. King, Electromagnetic engineering, app. 2, pp. 510 to 518 (McGraw-Hill Book Co., Inc., New York, N.Y., 1945).

[2] Ronold King, Electromagnetic engineering, Ch. I, Sec. 24, pp. 77 to 81, especially equation 13 (McGrawHill Book Co., New York, N.Y., 1956).

[3] R. W. P. King, Theory of linear antennas, Ch. II (Harvard University Press, Cambridge, Mass., 1956).

[4] Ronold King, Linear arrays: Currents, impedances, and fields, I. (Harvard University, Cambridge, Mass., May 1, 1959) Trans. IRE AP-7, 5440 (1959).

[5] Tai Tsun Wu and Ronold King, Driving point and input admittance of linear antennas, J. Appl. Phys. 30, 74 (1959).

[6] The logic of this step is made clear by a study of sections 2 and 4 of the paper, The cylindrical antenna: current and impedance, by Ronold King and David Middleton, Quart. Appl. Math. 4 (July 1946). An alternative reference is sec. $20 \mathrm{in} \mathrm{ch}$. II of R. W. P. King, Theory of linear antennas (Harvard University Press, Cambridge, Mass., 1956).

[7] R. W. P. King, Theory of linear antennas, p. 168 (Harvard University Press, Cambridge, Mass., 1956).

[8] R. W. P. King, Theory of linear antennas, ch. II, sec. 38 (Harvard University Press, Cambridge, Mass., 1956).

[9] Marcel Nicolet, Collision frequency of electrons in the terrestrial atmosphere, Phys. Fluids 2, 95 (1959).

[10] R. A. DiTaranto and J. J. Lamb, The space environment-a preliminary study, Elec. Manuf. (Gage Publishing Co., Oct. 1958). 


\subsection{Additional References}

A. Baños, Jr., and J. P. Wesley, The horizontal electric dipole in a conducting half-space, pt. I (1953), pt. II (1954), Univ. Calif. Marine Phys. Lab., Scripps Inst. Oceanog. (unpublished).

C. W. Harrison, Jr., and D. H. Denton, Jr., Impedance of a dipole in an ionized medium-I, Sandia Corp., New Mexico (1959) (unpublished).

C. W. Harrison, Jr., and D. H. Denton, Jr., Impedance of a dipole in an ionized medium-II, Sandia Corp., New Mexico (1959) (unpublished).

C. W. Harrison, Jr., Calculation of the impedance of a cylindrical antenna in an ionized medium of low attenuation factor by means of tables of the exponential integral for complex arguments, Sandia Corp., New Mexico (1959) (unpublished).

M. S. Macrakis, Cylindrical antenna immersed in a dissipative medium (input impedance), Cruft Lab., Harvard Univ. (1957) (unpublished).

R. K. Moore, The theory of radio communication between submerged submarines, Ph. D. Thesis, Graduate School of Cornell Univ. (June 1951) (unpublished).

C. T. Tai, Radiation of a Hertzian dipole immersed in a dissipative medium, Cruft Lab., Harvard Univ. (1947) (unpublished).

C. T. Tai, On radiation and radiating systems in the presence of a dissipative medium, Cruft Lab., Harvard Univ. (1949) (unpublished).

J. R. Wait and L. L. Campbell, The fields of an electric dipole in a semi-infinite conducting medium, J. Geophys. Research 58, 21 (1953).

J. R. Wait, The radiation fields of a horizontal dipole in a semi-infinite dissipative medium, J. Appl. Phys. 24, 958 (1953).

J. R. Wait and L. L. Campbell, Fields of dipoles in a semi-infinite conducting medium, J. Geophys. Research 58, 21 to 28,167 to 178 (1953).

J. R. Wait, Insulated loop antenna immersed in a conducting medium, J. Research NBS 59, 133 (1957) RP2781.

J. R. Wait, Radiation from a small loop immersed in a semi-infinite conducting medium, Can. J. Phys. 37, 672 (1959).

(Paper 64D4-70) 